\title{
Potato Fermented Fertilizer Modulates Soil Nitrification by Shift Niche of Functional Microorganisms to Increase Yield in North China
}

Qichun Zhang ( $\sim$ qczhang@zju.edu.cn )

Zhejiang University

\section{Longda Gong}

Touqeer Abbas

Dan Wu

Dan He

Hongjie Di

\section{Research Article}

Keywords: Potato Fermented Fertilizer, Soil microbial community, Comammox, AOA, AOB

Posted Date: February 17th, 2022

DOI: https://doi.org/10.21203/rs.3.rs-1341972/v1

License: (c) (i) This work is licensed under a Creative Commons Attribution 4.0 International License. Read Full License 


\section{Abstract}

\section{Aims}

Potato starch wastewater contains higher contents of essential nutrients, which can be feritilizer to help crop growth. However, the effects of potato fermented fertilizer on soil ecology and soil microbial community structure have not yet been elucidated. The objective of this study was to investigate the shifts of active ammonia oxidation microbial communities under different fertilization in a typical soil from North China.

\section{Methods}

The different levels of potato fermented fertilizer without or with chemical fertilizer were designed by field experiment.

\section{Results}

The results showed that the application of potato fermented fertilizer could significantly increase crop yields by 165$399 \%$ compared to Control. The content of available soil nutrients and the activity of saccharase and cellulase were increased when potato fermented fertilizer was applied, and the combination fertilizers further increased the content of Olsen-P by $145.6-166.7 \%, \mathrm{NO}_{3}{ }^{-}$by $15.2-81.1 \%$, Total $\mathrm{C}$ by $13.8-14 \%$, and Total $\mathrm{N}$ by $27.2-34.7 \%$ compared with potato fermented fertilizer (PW) treatments. Furthermore, the fermented potato fertilizer significantly stimulated the diversity of

soil microbial community, and increased the differentiation and stability of soil microbial networks in deep soils. Finally, the change of niche of soil Comammox(COM), ammonia-oxidizing archaea (AOA), and ammonia-oxidizing bacteria (AOB) was found after PW treatments, showed a significant positive correlation between AOA and COM $(r=0.79, P<$ $0.01), A O B$ and NOB $(r=0.7, P<0.05)$ instead of theoretically the competitive relationship between AOA and COM.

\section{Conclusions}

Potato fermented fertilizer modulates soil nitrification strategy by change the niche of soil functional microorganisms to increase fast-acting nutrients and increase crop yield.

\section{Introduction}

Potato (Solanum tuberosum L.), a crop with high nutritional and industrial values, is cultivated on massive agric soils worldwide. Potato is the largest non-cereal food crop worldwide and is the fourth most important food crop after wheat, corn, and rice. About $50 \%$ of all cultivated potatoes are used in processing industries such as potato starch and feed processing industries (Wang et al., 2009). In the potato starch industry, it has been estimated that $7 \mathrm{~m}^{3}$ of potato wastewater is produced during the processing of 1-ton potatoes. Subsequently, the potato wastewaters are treated through hot coagulation to remove about $90 \%$ of the protein to get deproteinized potato wastewater (Miedzianka et al., 2014).

The potential application of deproteinized potato wastewater in the field has numerous benefits. Firstly, potato waste contains abundant protein and amino acids. Notably, the decomposition of amino acids into more minor soluble compounds is critical in the terrestrial nitrogen cycle because it can provide an abundant source of nitrogen compounds and mineral substances for soil microorganisms (Noll et al., 2019). Moreover, potato wastewater contains significant amounts of mineral compounds (approximately 1\%), dominated by potassium and phosphorus (Kot et al., 2020). Over 
the years, studies have been conducted to assess the effects of potato wastewater as fertilizer on soil nutrients and crop yields, but has not been done the effects of potato wastewater as fertilizer on soil nutrient transformation and soil functional microorganisms.

Nitrogen $(\mathrm{N})$ is one of the essential nutrients for plants, and soil microorganisms can transform soil $\mathrm{N}$ into a usable form for plants through the catalytic nitrification process. Nitrification was long considered a two-step process involving ammonia to nitrite, followed by oxidation of nitrite to nitrate. It is worth noting that ammonia oxidation is catalyzed by ammonia-oxidizing microorganisms, including ammonia-oxidizing bacteria (AOB) and ammonia-oxidizing archaea (AOA) (Nunes-Alves, 2016; Abbas et al., 2020). Since the discovery of AOA, the contribution of AOA and AOB to ammonia oxidation and the ecological niches of $A O A$ and $A O B$ have been the research hotspots. However, to date, there is still controversy about the metabolic mode of AOA. The traditional view is that both AOA species are strictly autotrophic microorganisms (Kim et al., 2016). However, it has been widely documented that organic carbon is an important environmental factor affecting the AOA community. For example, the abundance of AOA still increased when the nitrification activity was completely inhibited by acetylene, suggesting that AOA may have heterotrophic activity (Prosser and Nicol, 2008; Jia and Conrad, 2009). Liu et al. (2018) also revealed that long-term manure application increased AOA abundance in some soils. The recent discovery of comammox bacteria (complete ammonia oxidizers) has also challenged the conventional theory of two-step nitrification (Daims et al., 2015a; Van Kessel et al., 2015). The high frequency of comammox bacteria was later reported in various artificial and natural habitats such as soil, rivers, sediments, drinking water treatment plants, and a range of wastewater treatment bioreactors, which suggests that comammox bacteria play a vital role in the $\mathrm{N}$ cycle (Cotto et al., 2020). The discovery of comammox bacteria led to a series of questions: (i) how is comammox regulated by environmental factors (ii) Is there niche differentiation of comammox, $\mathrm{AOA}$, and $\mathrm{AOB}$, and (iii) How does the discovery of comammox change our perception of the global $\mathrm{N}$ cycle (Santoro, 2016). The answer to the third question is based on the previous two questions. Currently, several studies have been conducted to answer the first question. For example, Liu et al. (2020b) found that comammox Nitrospira, including clade $A$ and clade $B$, contributed more to nitrifiers abundance in typical oligotrophic environments with a higher $\mathrm{pH}$ and lower temperature, particularly clade $A$ in the plateau and clade B in mountain and foothill areas of the upper reach. In addition, Xu et al. (2020) reported that the growth of comammox Nitrospira favors slightly alkaline soil with relatively high $\mathrm{C} / \mathrm{N}$ and low ammonia conditions. However, there are only a few studies on the respective roles of $A O A, A O B$, and comammox in nitrification and their association with each other under organic and inorganic fertilizers.

The objective of this study was to investigate the shifts of active ammonia oxidation microbial communities under different fertilization in a typical soil from North China by field experiments. Moreover, we investigated the effects of different levels applications of fermented potato fertilizer without or with chemical fertilizer on crop yield, soil physicochemical properties, enzyme activity, and niche differentiation of comammox, AOA, and AOB

\section{Materials And Methods}

\section{Experimental site}

The experiment was conducted in Pingjibao Experimental Greenhouse, Guyuan, Ningxia, China (N 35 $45^{\prime} 54^{\prime \prime}$, E $106^{\circ} 40^{\prime}$ 29") (Fig. S1), an area that belongs to a temperate continental climate. The annual average temperature was $8.5^{\circ} \mathrm{C}$, while the average precipitation was $450 \mathrm{~mm}$. The soil of the study area is sierozem with a weak humus accumulation process, low organic matter content and high calcium accumulation, and a sandy texture. The basic chemical properties of the soil are as $\mathrm{pH} 7.76$, Olsen-P $93.3 \mathrm{mg} \mathrm{kg}^{-1}$, total C $0.25 \%$, total $\mathrm{N} 0.04 \%$, and electrical conductivity $1015 \mu \mathrm{sm} \mathrm{cm}^{-1}$.

\section{Field experimental design}


The potato fermented fertilizer was made of fermenting potato starch wastewater using a strain of Serratia Marcescens Sakuensis NR screened from the soil (Zhou et al., 2020) at $15-17^{\circ} \mathrm{C}$ for 21 days. The chemical composition of the potato fermented fertilizer was showed in Table S1 and meet the standard of irrigation water quality (GB 5084 - 2021). The field experiment was conducted from 11th February to 7th May 2019. The planted crop was celery, and the variety was queen. The area of the field experiment was divided into 21 plots, with each plot covering $8.4 \mathrm{~m}^{2}(2 \mathrm{~m}$ wide $\times 4.2 \mathrm{~m}$ long). A randomized block design was used with three replicates. The fertilizer treatments were as follows: 1) CK, no fertilizer; 2) PW1, potato fermented fertilizer $3750 \mathrm{~kg} \bullet \mathrm{ha}^{-1}$; 3) PW2, potato fermented fertilizer $7500 \mathrm{~kg} \bullet \mathrm{ha}^{-1}$; 4) PW3,

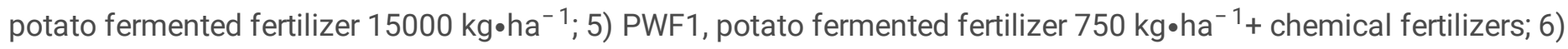

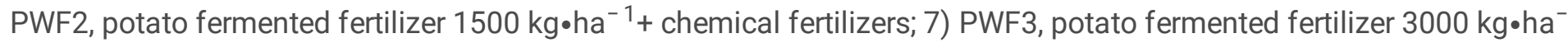

${ }^{1}+$ chemical fertilizers. The chemical fertilizers (urea, calcium superphosphate, and potassium sulfate) were applied as 450,150 , and $300 \mathrm{~kg} \bullet \mathrm{ha}^{-1}$ for $\mathrm{N}, \mathrm{P}$, and $\mathrm{K}$, respectively before celery was planted. The potato fermented fertilizer were applied ten times through drip irrigation, with the same amount being applied each time. Adjacent plots were separated by a ridge covered with plastic film to prevent seepage and inter-plot movements, and separate irrigation and drainage ditches were provided.

The yield of celery was measured after celery harvest. In addition, five soil core samples from 0-20 cm (topsoil) and 20-40 cm (deep soil) were collected from each plot on 7th May 2019 (after celery harvest). The samples were thoroughly mixed, and the pooled samples were transported on ice to the laboratory within 2-6 h of collection. One subsamples were air-dried, ground, and sieved through a $2 \mathrm{~mm}$ sieve to determine soil properties and soil enzyme activity, while the remaining soil was stored at $-80^{\circ} \mathrm{C}$ for DNA extraction.

\section{Determination of soil properties and enzyme activity}

The soil $\mathrm{pH}$ and conductivity were measured using a $\mathrm{CaCl}_{2}$ solution = 1:2.5 (v/v) suspension with a digital $\mathrm{pH}$ meter and conductivity meter. The content of exchangeable $\mathrm{NH}_{4}{ }^{+}$was measured using the indophenol blue colorimetric method, while $\mathrm{NO}_{3}{ }^{-}$concentration was measured using the $\mathrm{KCl}$ extraction for the colorimetric method. Soil available $\mathrm{P}(\mathrm{Olsen}-\mathrm{P}$ ) was determined using the $0.5 \mathrm{~mol} \cdot \mathrm{L}^{-1} \mathrm{NaHCO}_{3}$ extraction, molybdenum antimony anticolorimetric method. On the other hand, soil available $\mathrm{K}$ was determined using the $\mathrm{NH}_{4} \mathrm{OAC}$ extraction, flame photometry method. The soil was soaked in $0.1 \mathrm{~mol} \cdot \mathrm{L}^{-1} \mathrm{HCl}$, followed by drying and determining total carbon (TC) and total nitrogen (TN) using the elemental analyzer. The soil urease activity was performed using sodium phenate-sodium hypochlorite colorimetry, while soil saccharase and cellulase activities were measured using 3,5-dinitrosalicylic acid colorimetry. Furthermore, the soil catalase activity was measured using potassium permanganate titration described by Cai et al. (2019) and Yu et al. (2019).

\section{Soil DNA extraction, PCR, and MiSeq sequencing}

According to the manufacturer's instructions, soil DNA was extracted using OMEGA soil DNA kits. DNA quality was assessed using $0.8 \%$ agarose gel electrophoresis, and the DNA was quantified using a Nanodrop 2000 spectrophotometer. Next, the 16S rRNA variable V3-V4 region was amplified using the following primer pair: 515F (5'GTGYCAGCMGCCGCGGTAA-3') and 806R (5'-GGACTACHVGGGTWTCTAAT-3'). Polymerase chain reaction (PCR) was performed with a final volume of $25 \mathrm{ml}$ containing $5 \mathrm{ml} 5 \mathrm{X}$ reaction buffer, $5 \mathrm{ml} 5 \mathrm{X}$ GC buffer, $2 \mathrm{ml} \mathrm{dNTP}(2.5 \mathrm{mM}), 1 \mathrm{ml}$ forward primer $(10 \mathrm{mM}), 1 \mathrm{ml}$ reverse primer $(10 \mathrm{mM}), 2 \mathrm{ml}$ DNA template, $8.75 \mathrm{ml} \mathrm{ddH}_{2} \mathrm{O}$, and $0.25 \mathrm{ml}$ Q5 DNA polymerase. The amplification conditions included the following steps: initial denaturation at $98^{\circ} \mathrm{C}$ for 2 min; 30 cycles of denaturation at $98^{\circ} \mathrm{C}$ for $15 \mathrm{~s}$, annealing at $55^{\circ} \mathrm{C}$ for $30 \mathrm{~s}$, and extension at $72^{\circ} \mathrm{C}$ for $30 \mathrm{~s}$; and final extension at $72^{\circ} \mathrm{C}$ for $5 \mathrm{~min}$, followed by a hold at $10^{\circ} \mathrm{C}$. Each sample was amplified several times, and the PCR products were purified after mixing. Finally, the purified PCR product was subjected to a double terminal sequenced analysis using the Illumina MiSeq platform. 


\section{Quantitative PCR}

The copies of all the functional genes assessed in this experiment were estimated using quantitative real-time PCR (qPCR) in a StepOnePlus real-time PCR system (Applied Biosystems). The PCR mixture contained 10 $\mu$ l of QuantiTect SYBR ${ }^{\circledR}$ Green master mix (Qiagen), $0.5 \mu$ of forward and reverse primer each $(10 \mu \mathrm{M})$, and $1 \mu$ l of DNA template ( $5 \mathrm{ng} \mu \mathrm{I}^{-}$

${ }^{1}$ ) in a final volume of $20 \mu \mathrm{l}$. A melt curve analysis was conducted to verify the nonspecific amplification by increasing the temperature from 60 to $95^{\circ} \mathrm{C}$ with $4.4^{\circ} \mathrm{C}$ increments every second. Calculation of gene copies in the samples was based on a standard curve generated using standard plasmid DNA, which was made by cloning the target gene fragment as described by Bae et al. (2018). Table S2 shows the primers and conditions for each functional gene.

\section{Statistical analysis}

One-way analysis of variance (ANOVA) was used to determine the difference in soil parameters, bacterial community composition, and functional gene copies (Tukey, $n=3$ ), and the results were displayed using Origin 2020. Principal coordinate analysis (PCOA) was used to visualize the dissimilarity of beta diversity based on the Bray-Curtis distance of microbial community profiles. Differentially abundant OTUs were detected using the DESeq2 package, and the graph was displayed using ggrepel and ggplot2 packages in R. Network analysis was further performed to describe the complex co-occurrence pattern in different treatments, with the relative abundance of OTUs as nodes. Moreover, the results were constructed using W.G.C.N.A., igraph, and RMThreshold package in $\mathrm{R}$, while the graph was displayed using the Gephi version 0.9.2. Structural equation models are constructed by SPSS Amos. Finally, the original sequence data were deposited in the Genome Sequence Archive with accession number PRJNA727061.

\section{Results}

\section{Potato fermented fertilizer affected soil properties and soil enzyme activity}

An increase in the application level of potato fermented fertilizer boosts the yield of celery by $165.3-399.4 \%$ compared with CK (Table 1). The results showed a significant difference between PWF1, PWF2, PWF3, and PW1, PW2, PW3. This indicates that potato fermented fertilizer plus chemical fertilizer significantly increased celery yield compared to potato fermented fertilizer. Application of potato fermented fertilizer (PW1, PW2, PW3) decreased the total carbon (TC) of top and deep soils by $40.9-50 \%$ and total nitrogen ( $\mathrm{TN}$ ) by $40-57.8 \%$, compared with $\mathrm{CK}$. In addition, soil $\mathrm{NH}_{4}{ }^{+}$content in deep soils decreased by $54.3-58 \%$ whereas soil $\mathrm{NO}_{3}{ }^{-}$content increased by $25.6-109.4 \%$, compared with the levels in the CK (Table 1). Top and deep soils available phosphorus (Olsen-P) and potassium (Av-K) increased, whereas electrical conductivity (EC) decreased gradually with an increase in the level of potato fermented fertilizer. Application of potato fermented fertilizer with chemical fertilizer (PWF1, PWF2, PWF3) did not change the trend of potato fermented fertilizer effects on soil physical and chemical properties. However, the combination fertilizers further increased the content of topsoil Olsen-P by $145.6-166.7 \%$, top and deep soil $\mathrm{NO}_{3}{ }^{-}$by $15.2-81.1 \%$, TC by $13.8-14 \%$, and TN by $27.2-34.7 \%$ compared with PW1, PW2, PW3 treatments (Table 1). 
Table 1

Soil chemical properties after treatments

\begin{tabular}{|c|c|c|c|c|c|c|c|c|c|c|c|}
\hline Depth & Treatment & $\mathrm{pH}$ & $\begin{array}{l}\text { EC } \\
\text { ( } \mu s \\
\mathrm{~cm}^{-} \\
\left.{ }^{1}\right)\end{array}$ & $\begin{array}{l}\mathrm{NH}_{4}^{+} \\
(\mu \mathrm{g} \\
\mathrm{kg}^{-} \\
\left.{ }^{1}\right)\end{array}$ & $\begin{array}{l}\mathrm{NO}_{3}^{-} \\
(\mathrm{mg} \\
\left.\mathrm{kg}^{-1}\right)\end{array}$ & $\begin{array}{l}\text { Olsen- } \\
P \\
(\mathrm{mg} \\
\left.\mathrm{kg}^{-1}\right)\end{array}$ & $\begin{array}{l}\text { Av-K } \\
(\mathrm{mg} \\
\left.\mathrm{kg}^{-1}\right)\end{array}$ & $\begin{array}{l}\mathrm{FC} \\
(\%)\end{array}$ & $\begin{array}{l}\text { TC } \\
(\%)\end{array}$ & $\mathrm{TN}(\%)$ & $\begin{array}{l}\text { yield } \\
(\mathrm{kg} \\
\left.\mathrm{ha}^{-1}\right)\end{array}$ \\
\hline \multirow[t]{8}{*}{ Topsoil } & CK & $\begin{array}{l}7.83 \\
\pm \\
0.02 a\end{array}$ & $\begin{array}{l}1114 \\
\pm \\
104 \mathrm{~b}\end{array}$ & $\begin{array}{l}52.2 \\
\pm 5 a\end{array}$ & $\begin{array}{l}1.76 \pm \\
0.3 a\end{array}$ & $\begin{array}{l}42.3 \\
\pm \\
2.9 \mathrm{bc}\end{array}$ & $\begin{array}{l}92.2 \\
\pm 6.1 \mathrm{c}\end{array}$ & $\begin{array}{l}28.9 \\
\pm \\
1.4 \mathrm{~b}\end{array}$ & $\begin{array}{l}0.22 \\
\pm \\
0.05 a\end{array}$ & $\begin{array}{l}0.045 \\
\pm \\
0.002 a\end{array}$ & $\begin{array}{l}16020 \\
\pm 801 \mathrm{c}\end{array}$ \\
\hline & PW1 & $\begin{array}{l}7.76 \\
\pm \\
0.04 a\end{array}$ & $\begin{array}{l}1377 \\
\pm 73 a\end{array}$ & $\begin{array}{l}43.6 \\
\pm \\
6.2 a\end{array}$ & $\begin{array}{l}1.4 \pm \\
0.26 \mathrm{a}\end{array}$ & $\begin{array}{l}51.8 \\
\pm \\
9.2 \mathrm{ab}\end{array}$ & $\begin{array}{l}95.8 \\
\pm 3.7 \mathrm{c}\end{array}$ & $\begin{array}{l}36.3 \\
\pm 2 a\end{array}$ & $\begin{array}{l}0.13 \\
\pm \\
0.08 \mathrm{~b}\end{array}$ & $\begin{array}{l}0.025 \\
\pm \\
0.003 b\end{array}$ & $\begin{array}{l}42150 \\
\pm \\
2125 b\end{array}$ \\
\hline & PW2 & $\begin{array}{l}7.8 \pm \\
0.01 a\end{array}$ & $\begin{array}{l}1022 \\
\pm 29 b\end{array}$ & $\begin{array}{l}42.7 \\
\pm \\
3.1 \mathrm{a}\end{array}$ & $\begin{array}{l}2.1 \pm \\
0.65 \mathrm{a}\end{array}$ & $\begin{array}{l}38.6 \\
\pm 5.2 \mathrm{c}\end{array}$ & $\begin{array}{l}114.5 \\
\pm 3.1 \mathrm{~b}\end{array}$ & $\begin{array}{l}36.4 \\
\pm \\
1.4 \mathrm{a}\end{array}$ & $\begin{array}{l}0.11 \\
\pm \\
0.08 \mathrm{~b}\end{array}$ & $\begin{array}{l}0.023 \\
\pm \\
0.003 b\end{array}$ & $\begin{array}{l}43050 \\
\pm \\
2152 b\end{array}$ \\
\hline & PW3 & $\begin{array}{l}7.72 \\
\pm \\
0.03 a\end{array}$ & $\begin{array}{l}780 \\
\pm 17 \mathrm{c}\end{array}$ & $\begin{array}{l}48.6 \\
\pm \\
4.8 \mathrm{a}\end{array}$ & $\begin{array}{l}1.7 \pm \\
0.43 a\end{array}$ & $\begin{array}{l}60.1 \\
\pm 1.0 \mathrm{a}\end{array}$ & $\begin{array}{l}123.6 \\
\pm 1.3 a\end{array}$ & $\begin{array}{l}36.5 \\
\pm \\
1.3 a\end{array}$ & $\begin{array}{l}0.11 \\
\pm \\
0.05 \mathrm{~b}\end{array}$ & $\begin{array}{l}0.027 \\
\pm \\
0.003 \mathrm{~b}\end{array}$ & $\begin{array}{l}68406 \\
\pm \\
2420 a\end{array}$ \\
\hline & CK & $\begin{array}{l}7.83 \\
\pm \\
0.02 a\end{array}$ & $\begin{array}{l}1114 \\
\pm \\
104 a\end{array}$ & $\begin{array}{l}52.2 \\
\pm 5 a\end{array}$ & $\begin{array}{l}1.76 \pm \\
0.3 c\end{array}$ & $\begin{array}{l}42.3 \\
\pm 2.9 \mathrm{~b}\end{array}$ & $\begin{array}{l}92.2 \\
\pm 6.1 \mathrm{a}\end{array}$ & $\begin{array}{l}28.9 \\
\pm \\
1.4 \mathrm{c}\end{array}$ & $\begin{array}{l}0.22 \\
\pm \\
0.05 a\end{array}$ & $\begin{array}{l}0.045 \\
\pm \\
0.002 a\end{array}$ & $\begin{array}{l}16020 \\
\pm 801 c\end{array}$ \\
\hline & PWF1 & $\begin{array}{l}7.58 \\
\pm \\
0.02 b\end{array}$ & $\begin{array}{l}1037 \\
\pm \\
52 a b\end{array}$ & $\begin{array}{l}40.4 \\
\pm \\
6.3 a\end{array}$ & $\begin{array}{l}2.65 \pm \\
0.23 b\end{array}$ & $\begin{array}{l}147.8 \\
\pm \\
14.1 \mathrm{a}\end{array}$ & $\begin{array}{l}99.7 \\
\pm 6.5 \mathrm{a}\end{array}$ & $\begin{array}{l}40.1 \\
\pm \\
1.4 \mathrm{a}\end{array}$ & $\begin{array}{l}0.14 \\
\pm \\
0.03 \mathrm{~b}\end{array}$ & $\begin{array}{l}0.036 \\
\pm \\
0.003 b\end{array}$ & $\begin{array}{l}66645 \\
\pm \\
3420 \mathrm{~b}\end{array}$ \\
\hline & PWF2 & $\begin{array}{l}7.58 \\
\pm \\
0.01 b\end{array}$ & $\begin{array}{l}966 \\
\pm 16 \mathrm{~b}\end{array}$ & $\begin{array}{l}59.2 \\
\pm \\
3.1 \mathrm{a}\end{array}$ & $\begin{array}{l}3.03 \pm \\
0.39 b\end{array}$ & $\begin{array}{l}151.5 \\
\pm 12 a\end{array}$ & $\begin{array}{l}100.4 \\
\pm 8.5 a\end{array}$ & $\begin{array}{l}37.8 \\
\pm \\
2.9 a b\end{array}$ & $\begin{array}{l}0.13 \\
\pm \\
0.05 b\end{array}$ & $\begin{array}{l}0.034 \\
\pm \\
0.002 b\end{array}$ & $\begin{array}{l}67710 \\
\pm \\
3380 \mathrm{~b}\end{array}$ \\
\hline & PWF3 & $\begin{array}{l}7.58 \\
\pm \\
0.01 b\end{array}$ & $\begin{array}{l}1025 \\
\pm \\
10 a b\end{array}$ & $\begin{array}{l}45 \pm \\
4.8 \mathrm{a}\end{array}$ & $\begin{array}{l}3.72 \pm \\
0.14 a\end{array}$ & $\begin{array}{l}163.3 \\
\pm \\
18.2 \mathrm{a}\end{array}$ & $\begin{array}{l}90.7 \\
\pm 2.1 \mathrm{a}\end{array}$ & $\begin{array}{l}35.4 \\
\pm \\
1.2 \mathrm{bc}\end{array}$ & $\begin{array}{l}0.13 \\
\pm \\
0.04 \mathrm{~b}\end{array}$ & $\begin{array}{l}0.031 \\
\pm \\
0.002 b\end{array}$ & $\begin{array}{l}80004 \\
\pm \\
3217 a\end{array}$ \\
\hline \multirow[t]{6}{*}{$\begin{array}{l}\text { Deep } \\
\text { soil }\end{array}$} & CK & $\begin{array}{l}7.7 \pm \\
0.01 a\end{array}$ & $\begin{array}{l}916 \\
\pm 2 a\end{array}$ & $\begin{array}{l}81.5 \\
\pm \\
6.5 a\end{array}$ & $\begin{array}{l}1.17 \pm \\
0.04 \mathrm{~b}\end{array}$ & $\begin{array}{l}29.6 \\
\pm \\
0.8 \mathrm{ab}\end{array}$ & $\begin{array}{l}94.4 \\
\pm 3.8 \mathrm{c}\end{array}$ & $\begin{array}{l}40.1 \\
\pm \\
2.3 a\end{array}$ & $\begin{array}{l}0.24 \\
\pm \\
0.05 a\end{array}$ & $\begin{array}{l}0.042 \\
\pm \\
0.001 \mathrm{a}\end{array}$ & NA \\
\hline & PW1 & $\begin{array}{l}7.77 \\
\pm \\
0.02 a\end{array}$ & $\begin{array}{l}813 \\
\pm 17 b\end{array}$ & $\begin{array}{l}37.5 \\
\pm 7 b\end{array}$ & $\begin{array}{l}2.45 \pm \\
0.36 a\end{array}$ & $\begin{array}{l}32.4 \\
\pm \\
8.8 \mathrm{ab}\end{array}$ & $\begin{array}{l}94.7 \\
\pm 4.5 \mathrm{c}\end{array}$ & $\begin{array}{l}33.9 \\
\pm \\
2.1 \mathrm{~b}\end{array}$ & $\begin{array}{l}0.14 \\
\pm \\
0.09 \mathrm{~b}\end{array}$ & $\begin{array}{l}0.026 \\
\pm \\
0.001 b\end{array}$ & NA \\
\hline & PW2 & $\begin{array}{l}7.78 \\
\pm \\
0.2 \mathrm{a}\end{array}$ & $\begin{array}{l}753 \\
\pm 23 c\end{array}$ & $\begin{array}{l}39.9 \\
\pm \\
2.2 \mathrm{~b}\end{array}$ & $\begin{array}{l}1.47 \pm \\
0.11 b\end{array}$ & $\begin{array}{l}25.9 \\
\pm 2 b\end{array}$ & $\begin{array}{l}107.8 \\
\pm 1.6 \mathrm{~b}\end{array}$ & $\begin{array}{l}36.4 \\
\pm \\
3.1 \mathrm{~b}\end{array}$ & $\begin{array}{l}0.11 \\
\pm \\
0.05 b\end{array}$ & $\begin{array}{l}0.022 \\
\pm \\
0.002 b\end{array}$ & NA \\
\hline & PW3 & $\begin{array}{l}7.8 \pm \\
0.03 a\end{array}$ & $\begin{array}{l}711 \\
\pm 10 \mathrm{~d}\end{array}$ & $\begin{array}{l}38.4 \\
\pm \\
2.1 \mathrm{~b}\end{array}$ & $\begin{array}{l}1.94 \pm \\
0.88 \mathrm{ab}\end{array}$ & $\begin{array}{l}36.1 \\
\pm 2.3 a\end{array}$ & $\begin{array}{l}118.7 \\
\pm 3 a\end{array}$ & $\begin{array}{l}37.6 \\
\pm \\
2.2 \mathrm{~b}\end{array}$ & $\begin{array}{l}0.11 \\
\pm \\
0.0 .4 \mathrm{~b}\end{array}$ & $\begin{array}{l}0.019 \\
\pm \\
0.002 c\end{array}$ & NA \\
\hline & CK & $\begin{array}{l}7.7 \pm \\
0.01 a\end{array}$ & $\begin{array}{l}916 \\
\pm 2 a\end{array}$ & $\begin{array}{l}81.5 \\
\pm \\
6.5 a\end{array}$ & $\begin{array}{l}1.17 \pm \\
0.03 c\end{array}$ & $\begin{array}{l}29.6 \\
\pm 0.8 \mathrm{~b}\end{array}$ & $\begin{array}{l}94.4 \\
\pm 3.8 \mathrm{a}\end{array}$ & $\begin{array}{l}40 \pm \\
0.2 a\end{array}$ & $\begin{array}{l}0.24 \\
\pm \\
0.05 a\end{array}$ & $\begin{array}{l}0.042 \\
\pm \\
0.001 a\end{array}$ & NA \\
\hline & PWF1 & $\begin{array}{l}7.67 \\
\pm \\
0.01 a\end{array}$ & $\begin{array}{l}711 \\
\pm 6 c\end{array}$ & $\begin{array}{l}36.3 \\
\pm \\
4.8 \mathrm{~b}\end{array}$ & $\begin{array}{l}1.4 \pm \\
0.19 \mathrm{c}\end{array}$ & $\begin{array}{l}22.5 \\
\pm 2.6 \mathrm{c}\end{array}$ & $\begin{array}{l}94.5 \\
\pm 0.8 \mathrm{a}\end{array}$ & $\begin{array}{l}34.1 \\
\pm \\
1.4 \mathrm{c}\end{array}$ & $\begin{array}{l}0.13 \\
\pm \\
0.03 \mathrm{~b}\end{array}$ & $\begin{array}{l}0.024 \\
\pm \\
0.002 b\end{array}$ & NA \\
\hline
\end{tabular}




\begin{tabular}{|c|c|c|c|c|c|c|c|c|c|c|c|}
\hline Depth & Treatment & $\mathrm{pH}$ & $\begin{array}{l}\text { EC } \\
\text { ( } \mu s \\
\mathrm{~cm}^{-} \\
\left.{ }^{1}\right)\end{array}$ & $\begin{array}{l}\mathrm{NH}_{4}^{+} \\
(\mu \mathrm{g} \\
\mathrm{kg}^{-} \\
1)\end{array}$ & $\begin{array}{l}\mathrm{NO}_{3}^{-} \\
(\mathrm{mg} \\
\left.\mathrm{kg}^{-1}\right)\end{array}$ & $\begin{array}{l}\text { Olsen- } \\
\mathrm{P} \\
(\mathrm{mg} \\
\left.\mathrm{kg}^{-1}\right)\end{array}$ & $\begin{array}{l}\mathrm{Av}-\mathrm{K} \\
(\mathrm{mg} \\
\left.\mathrm{kg}^{-1}\right)\end{array}$ & $\begin{array}{l}\text { FC } \\
(\%)\end{array}$ & $\begin{array}{l}\mathrm{TC} \\
(\%)\end{array}$ & $\mathrm{TN}(\%)$ & $\begin{array}{l}\text { yield } \\
(\mathrm{kg} \\
\left.\mathrm{ha}^{-1}\right)\end{array}$ \\
\hline & PWF2 & $\begin{array}{l}7.73 \\
\pm \\
0.03 a\end{array}$ & $\begin{array}{l}785 \\
\pm 17 \mathrm{~b}\end{array}$ & $\begin{array}{l}34.3 \\
\pm \\
5.3 b\end{array}$ & $\begin{array}{l}2.95 \pm \\
0.23 a\end{array}$ & $\begin{array}{l}33.4 \\
\pm 4.7 \mathrm{~b}\end{array}$ & $\begin{array}{l}87.6 \\
\pm 2 a\end{array}$ & $\begin{array}{l}37.8 \\
\pm 1 a b\end{array}$ & $\begin{array}{l}0.14 \\
\pm \\
0.05 b\end{array}$ & $\begin{array}{l}0.031 \\
\pm \\
0.002 b\end{array}$ & NA \\
\hline & PWF3 & $\begin{array}{l}7.71 \\
\pm \\
0.02 a\end{array}$ & $\begin{array}{l}722 \\
\pm 7 c\end{array}$ & $\begin{array}{l}39.1 \\
\pm \\
6.5 \mathrm{~b}\end{array}$ & $\begin{array}{l}2.39 \pm \\
0.49 b\end{array}$ & $\begin{array}{l}46.3 \\
\pm 4.6 \mathrm{a}\end{array}$ & $\begin{array}{l}91.7 \\
\pm 0.5 a\end{array}$ & $\begin{array}{l}34.8 \\
\pm 1 \mathrm{bc}\end{array}$ & $\begin{array}{l}0.14 \\
\pm \\
0.05 \mathrm{~b}\end{array}$ & $\begin{array}{l}0.029 \\
\pm \\
0.002 b\end{array}$ & NA \\
\hline
\end{tabular}

Soil urease activity was not significantly affected by PW treatments (Fig. 1). An increase in the level of potato fermented fertilizer gradually increased the activity of saccharase and cellulase by $54.8-71.4 \%$ and $21.7-98.7 \%$ in PWF1, PWF2, PWF3 treatments compared with CK treatment. In addition, the activity of saccharase and cellulase increased by 11.933.3\% and 4.8-97.5\% after application of PW1, PW2, PW3 treatments, respectively, whereas catalase activity showed the opposite trend at both layers.

\section{Potato fermented fertilizer affected soil bacterial community and composition}

The 16S rRNA gene sequencing was performed to explore the effects of different levels and fertilizers treatments on soil microbial community composition. The alpha diversity of the soil bacteria community was characterized by Chao1, ACE, Simpson, and Shannon diversity indices (Table 2). The results showed that potato fermented fertilizer with chemical fertilizer (PWF1, PWF2, PWF3) significantly increased the richness index (Chao1 and ACE) and decreased the diversity index (Simpson and Shannon) of topsoil and deep soil compared with administration of CK. Moreover, principal coordinate analysis (PCoA) showed the contribution rate of the first row of PW1, PW2, PW3, CK and PWF1, PWF2, PWF3, CK treatments of deep soil (56.74\% and 54.3\%) were significantly higher compared with that of topsoil (27.6\% and $34.61 \%$ ) (Fig. 2). Application of potato fermented fertilizer was significantly correlated with the beta diversity of the soil bacteria community of top and deep soil in PW1, PW2, PW3, CK treatments $(R=0.76, P<0.01 ; R=0.66, P<0.01)$ and PWF1, PWF2, PWF3, CK treatments $(R=0.96, P<0.01 ; R=0.98, P<0.01)$. Moreover, applications of different levels of potato fermented fertilizer $(R=0.93, P<0.01$ in $P W 1, P W 2, P W 3$ treatments; $R=0.95, P<0.01$ in $P W F 1, P W F 2, P W F 3$ treatments) showed significant differences in beta diversity of the deep soil bacteria community. However, the analysis did not show a significant difference between PW1, PW2, PW3 treatments $(R=0.49, P=0.011)$, while a significant difference was observed in topsoil under PWF1, PWF2, and PWF3 treatments $(R=0.77, P<0.01)$. 
Table 2

Effects of potato fermented fertilizer on soil microbial community diversity

\begin{tabular}{|llllll|}
\hline Depth & Treatment & Chao1 & ACE & Simpson & Shannon \\
\hline Topsoil & CK & $4437.9 \pm 113.1 \mathrm{c}$ & $6173.3 \pm 129.2 \mathrm{~b}$ & $0.01 \pm 0.001 \mathrm{c}$ & $2.83 \pm 0.02 \mathrm{ab}$ \\
\cline { 2 - 6 } & PW1 & $5006.7 \pm 316.3 \mathrm{a}$ & $6798.1 \pm 332.7 \mathrm{a}$ & $0.0126 \pm 0.001 \mathrm{~b}$ & $2.86 \pm 0.02 \mathrm{a}$ \\
\cline { 2 - 6 } & PW2 & $4818.7 \pm 50.4 \mathrm{ba}$ & $6694.9 \pm 77.6 \mathrm{a}$ & $0.015 \pm 0.002 \mathrm{a}$ & $2.83 \pm 0.01 \mathrm{~b}$ \\
\hline & PW3 & $4554.9 \pm 145.7 \mathrm{bc}$ & $6305.2 \pm 34.3 \mathrm{~b}$ & $0.009 \pm 0.001 \mathrm{c}$ & $2.86 \pm 0.01 \mathrm{a}$ \\
\hline & CK & $4437.9 \pm 113.1 \mathrm{a}$ & $6173.2 \pm 129.2 \mathrm{ab}$ & $0.01 \pm 0.001 \mathrm{~b}$ & $2.83 \pm 0.02 \mathrm{a}$ \\
\hline PWF1 & $4265.6 \pm 61.3 \mathrm{a}$ & $6045.3 \pm 70.7 \mathrm{~b}$ & $0.014 \pm 0.002 \mathrm{a}$ & $2.78 \pm 0.02 \mathrm{~b}$ \\
\hline PWF2 & $4667 \pm 454.7 \mathrm{a}$ & $6546.8 \pm 417 \mathrm{a}$ & $0.015 \pm 0.002 \mathrm{a}$ & $2.83 \pm 0.02 \mathrm{a}$ \\
\hline PWF3 & $4356.3 \pm 152.7 \mathrm{a}$ & $6110.2 \pm 142.5 \mathrm{ab}$ & $0.013 \pm 0.001 \mathrm{a}$ & $2.79 \pm 0.01 \mathrm{~b}$ \\
\hline CK & $4362.4 \pm 207.6 \mathrm{~b}$ & $6075.6 \pm 270.2 \mathrm{~b}$ & $0.01 \pm 0.001 \mathrm{~b}$ & $2.8 \pm 0.02 \mathrm{~b}$ \\
\hline PW1 & $4876.6 \pm 281.4 \mathrm{a}$ & $6786.1 \pm 299.2 \mathrm{a}$ & $0.02 \pm 0.004 \mathrm{a}$ & $2.78 \pm 0.04 \mathrm{~b}$ \\
\hline PW2 & $4940.8 \pm 61.4 \mathrm{a}$ & $6839.4 \pm 118.5 \mathrm{a}$ & $0.02 \pm 0.003 \mathrm{a}$ & $2.8 \pm 0.04 \mathrm{~b}$ \\
\hline PW3 & $5106.3 \pm 82.8 \mathrm{a}$ & $6924.9 \pm 129.5 \mathrm{a}$ & $0.01 \pm 0.001 \mathrm{~b}$ & $2.87 \pm 0.01 \mathrm{a}$ \\
\hline CK & $4362.4 \pm 207.6 \mathrm{c}$ & $6075.6 \pm 270.2 \mathrm{~b}$ & $0.01 \pm 0.001 \mathrm{~b}$ & $2.8 \pm 0.02 \mathrm{~b}$ \\
\hline PWF1 & $4684.7 \pm 44.8 \mathrm{~b}$ & $6581.9 \pm 63.9 \mathrm{a}$ & $0.018 \pm 0.001 \mathrm{a}$ & $2.8 \pm 0.01 \mathrm{~b}$ \\
\hline PWF2 & $4309.8 \pm 128.7 \mathrm{c}$ & $6102.9 \pm 251 \mathrm{~b}$ & $0.017 \pm 0.002 \mathrm{a}$ & $2.78 \pm 0.02 \mathrm{c}$ \\
\hline
\end{tabular}

Operational taxonomic units (OTUs) are showed in Fig. 3 which generated based on similarity of mainly clustered PCR amplified 16S sequences. Application of potato fertilizer treatments significantly upgraded or downgraded OTUs, including amounts and kinds of different OTUs compared with CK (Fig. 3). However, the application of potato fermented fertilizer significantly increased the abundance of Arthrobacter(OTU1), Pseudomonas (OTU83), and Azoarcus (OTU32), which are involved in carbon mineralization and N fixation (Karigar et al., 2006; Chevalier et al., 2017; Sperfeld et al., 2018). On the contrary, application of potato fermented fertilizer significantly decreased the abundance of Nitrosospira (OTU34), Nitrolancea (OTU518), and Aquicella (OTU1227) (Fig S2), which are nitrifying and pathogenic bacteria (Santos et al., 2003; Norton et al., 2008; Spieck et al., 2020). Effects on different OTUs in all treatments at topsoils were similar (Fig. 3a-f). The number, type, and degree of difference OTUs increased gradually with increased levels of potato fermented fertilizer at deep soils (Fig. 3g-i). However, the number, type, and degree of difference of OTUs in PWF1, PWF2, PWF3 treatments were similar to PW3 treatment, which was the highest compared with PW1 and PW2 treatments (Fig. 3j-I). Covariance network analysis shows that applying potato fermented fertilizer decreases the number of nodes, edges, modularity and average clustering coefficient of the network, and increases the weighted average degree and the proportion of positively correlated edges (Fig. 4). The addition of fertilizer continued to exacerbate these changes in the network. This suggests that the addition of potato fermented fertilizer reduces the diversity of soil microorganisms, causing the microbial community to evolve in a direction that is more suitable for the environment in which potato fermented fertilizer is added, and reduces the competitive relationships in the community making it more stable. These results indicate that the application of potato fermented fertilizer significantly affected soil microorganisms and microbial communities in deep soil whatever applied with or without chemical fertilizer. Moreover, chemical fertilizer can improve the effect of potato fermented fertilizer on the soil microbial community. 


\section{Potato fermented fertilizer modulates soil nitrification process}

The above results showed that $\mathrm{NO}_{3}{ }^{-}$the content of topsoil was significantly increased after increased application PWF levels but not in PW levels (Table 1), indicates that chemical fertilizer can affect the conversion of organic $\mathrm{N}$ to inorganic $\mathrm{N}$ and the nitrification process. Therefore, the abundance of functional genes of essential microorganisms implicated in the nitrification process, including ammonia-oxidizing archaea (AOA), ammonia-oxidizing bacteria (AOB), comammox Nitrospira clade $A$ and $B$, and nitrite-oxidizing bacteria (NOB) were explored (Fig. 5). The results showed that the abundance of $\mathrm{AOB}$ and $\mathrm{NOB}$ decreased with the increase level of potato fermented fertilizer. It is interesting that a significant increase in abundance of AOA and comammox (COM) was observed in PW1, PW2, PW3, and PWF1, PWF2, PWF3 treatments at deep soils, but the effect was not significant for the application of PW1, PW2, PW3 treatments on topsoil. The abundance of AOA significantly decreased, and COM significantly increased at the topsoil after applying PWF1, PWF2, PWF3 treatments. These results suggested that potato fermented fertilizer significantly affected the microbial community in deep soil in this study.

Correlations between $\mathrm{N}$ cycling functional genes, environmental factors, and input of potato fermented fertilizer (Input) in deep soils were further explored (Fig. 6). The results showed a significant positive correlation between copies of AOA and COM $(r=0.79, P<0.01), A O B$, and NOB $(r=0.7, P<0.05)$ in PW1, PW2, PW3 treatments (Fig. 6a). Furthermore, a significant positive correlation was observed between copies of AOA, COM, and Input $(r=0.72, P<0.01 ; r=0.67, P<0.05)$ but not in AOB, NOB, and Input. In PWF1, PWF2, PWF3 treatments (Fig. 6b), a significant positive correlation was observed between NOB and AOB $(r=0.64, P<0.05)$, Input and COM $(r=0.69, P<0.05)$, while no correlation was observed between $A O A$ and $C O M, A O A$ and Input. A significant negative correlation was observed between $A O A$ and $A O B(R=-0.81$, $P<0.01)$. Based on the correlation between potato fermented fertilizer application and AOA and comammox, we further analysed their relationship with yield by means of structural equation modelling, which indicated that potato fermented fertilizer application increased the nitrate-nitrogen content of the soil by increasing the abundance of comammox, while potato fermented fertilizer application also increased the microbial load of the soil, which increases led to an increase in celery yield (Fig. 7).

\section{Discussion}

\section{The fermented potato fertilizer changed soil properties and enzyme activity}

Potato wastewater contains significant amounts of mineral compounds (approximately $1 \%$ ), which are dominated by potassium (K) and phosphorus $(\mathrm{P})$, and proteins, including patatin, alkaline inhibitors of proteases, and complex $22 \mathrm{kDa}$ proteins (Markiewicz et al., 2015). Kurcz et al. (2016) reported that glutamic and aspartic acid's highest share of amino acids is taken up. Therefore, the application of potato fermented fertilizer significantly increases soil Olsen-P and Av-K at the topsoil and deep soil. Notably, P is easily fixed in alkaline soil, while $\mathrm{K}$ is easily lost in alkaline soil, which can be attributed to the fact that the fluidity of $P$ and $K$ in soil is very different. Thus, most of the P in PWF1, PWF2, and PWF3 treatments were fixed on the surface, while most of the $\mathrm{K}$ was lost during irrigation, which explains why the soil Av- $\mathrm{K}$ was lower than PW1, PW2, and PW3 treatments.

The study site was Yinchuan, Ningxia, China, an area with sierozem soil with a weak humus accumulation, low organic matter content, and calcium accumulation, indicating that the soil is poor. Results showed that the soil TC content decreased when potato fermented fertilizer was applied without chemical fertilizer (PW1, PW2, and PW3 treatments) but increased significantly in PWF1, PWF2, and PWF3 treatments compared to the PW1, PW2, and PW3 treatments.

Fontaine et al. (2010) reported that the supply of fresh carbon might accelerate soil carbon decomposition and induce a

Page 9/18 
negative carbon balance because of the lack of energy available to soil microorganisms. Moreover, Li et al. (2019a) reported that short-term $\mathrm{N}$-addition could significantly increase the soil respiration and its components, $\mathrm{C}$ and $\mathrm{N}$, storage in soil. Therefore, potato fermented fertilizer with chemical fertilizer increased the content of nutrients that are rapidly utilized by microorganisms, thereby accelerating the utilization of potato fermented fertilizer by soil microorganisms and increasing the storage of soil carbon and nitrogen.

Previous studies have indicated that soil urease, saccharase, cellulase, and catalase are associated with the soil health, carbon cycle, and antioxidant defense system (Zhang et al., 2014; Kaya et al., 2019; Liu et al., 2020a). In this study, there was no significant change of urease in each treatment, suggesting that potato fermented fertilizer has no adverse effects on soil biochemical functions. Liang et al. (2018) reported that potato fermented fertilizer contains a large amount of soluble organic carbon, stimulating the soil carbon cycle (activity of saccharase and cellulase) when applied to the soil. At the same time, the decomposition ability of soil to hydrogen peroxide (the activity of catalase) was decreased after the input of organic carbon (Fig. 1), which is consistent with the results reported by Bissey et al. (2006). The increase in the activity of soil saccharase and cellulase in PWF1, PWF2, and PWF3 treatments was more significant than that in PW1, PW2, and PW3 treatments, indicating that potato fermented fertilizer with chemical fertilizer has a more vital ability to decompose organic matter and sequester carbon than potato fermented fertilizer alone. Soil available $\mathrm{N}$ is the crucial factor affecting soil carbon cycle and priming effect ( $\mathrm{Li}$ et al., 2018), and the addition of $\mathrm{P}$ stimulates soil organic matter priming by inducing microbial demand for $\mathrm{N}$ and stimulating the growth of soil organic matter degrading populations (Mehnaz et al., 2019). Therefore, it can be concluded that the available nutrients in the chemical fertilizer can induce soil microorganisms to utilize potato fermented fertilizer fully.

\section{The fermented potato fertilizer changed the soil microbial community structure in deep soils}

Results showed that potato fermented fertilizer significantly increased the richness of the soil microbial community (Table 2). However, the diversity of the soil microbial community was decreased, which might have been caused by the decrease of soil catalase activity. This can be attributed to the fact that decreased catalase activity results in the accumulation of $\mathrm{H}_{2} \mathrm{O}_{2}$ in soil, which is highly toxic to many groups of microorganisms in the soil (Kakosová et al., 2017), ultimately leading to the decrease of soil microbial diversity. The results of soil microbial richness index, PCoA, differential OTUs, and topological properties of network analysis suggested that application of potato fermented fertilizer could significantly change the structure of soil microbial community, and the changes were more significant in deep soils. This can be explained by the potato fermented fertilizer infiltrating faster to deep soils when it was applied to the surface soil due to the sandy soil of research area. Thus, the potato fermented fertilizer had a more significant effect on deep soils. Moreover, the results indicated that the potato fermented fertilizer, especially with chemical fertilizer, can significantly differ between different levels (Fig. 2) and stimulate differentiation of OTUs (Fig. 3). It can also significantly increase the network edge, node, network fragmentation and stability (Fig. 4). These results further confirmed the above conclusion that available nutrients in chemical fertilizers can induce soil microorganisms to utilize potato fermented fertilizer fully.

Several previous studies have reported that application of organic and inorganic fertilizers can influence the soil biochemical attributes, soil microbial community composition and abundance, increase soil bacterial and fungi community, to build a stable soil environment, and inhibit the occurrence of plant diseases compared to the application of organic or inorganic fertilizers alone (Leite et al., 2017; Ali et al., 2019; Li et al., 2019b; Tang et al., 2020). Most soil microorganisms can directly utilize the available $\mathrm{N}$ and $\mathrm{P}$ from fertilizers, including autotrophic, heterotrophic, and mixotrophic microorganisms that can use organic carbon and $\mathrm{CO}_{2}$ as carbon sources. However, microorganisms require much more $\mathrm{C}$ than $\mathrm{N}$ and $\mathrm{P}$. Therefore, when there are many available $\mathrm{N}$ and $\mathrm{P}$ in the soil system, soil microorganisms will be stimulated to decompose a large amount of organic carbon or fix $\mathrm{CO}_{2}$ in the air. This can explain why short-term

Page $10 / 18$ 
$\mathrm{N}$ fertilizers can significantly increase the soil respiration $\mathrm{C}$ storage in soil and shift from a more oligotrophic bacterial community to one that is more eutrophic (Fierer et al., 2012; Li et al., 2019a).

\section{The fermented potato fertilizer changed soil nitrification strategy by changing the niche of soil functional microorganisms}

Results indicated that the application of potato fermented fertilizer, especially with chemical fertilizer, can promote soil nitrification. The application of potato fermented fertilizer alone treatments shaped soil organic nutrient environment, while the application of potato fermented fertilizer with chemical fertilizer treatments shaped mixed (organic plus inorganic) nutrient environments. Therefore, we hypothesize that there are different strategies for soil nitrification in the above two nutrient environments. The results obtained in this study have shown that the abundance of AOA and COM increased in these two environments while the abundance of $A O B$ decreased. However, AOA and COM had positive correlations in the organic nutrient environment, and no correlation was observed in the mixed nutrient environment. The order of ammonium affinity for these three functional genes was $C O M>A O A>A O B$, which suggests that COM is more suitable for surviving in oligotrophic soils (Kits et al., 2017; Sakoula et al., 2020). It is worth noting that organic nutrient environments have a relatively low ammonium concentration because the ammonium is derived from the mineralization of organic matter, not directly from chemical fertilizer. Daims et al. (2015b) reported that both AOA and COM use ammonium as the substrate in $\mathrm{N}$ transformation. Therefore, in theory, there is a competitive relationship between AOA and COM; however, this study's results showed a significant positive correlation $(R=0.79, P<0.01)$ between the copies of AOA and COM. Thus, we proposed two hypotheses based on this result: (1) AOA directly utilizes the organic matter in potato fermented fertilizer. Multiple studies have shown that some AOA may have mixed or heterotrophic growth strategies (Prosser and Nicol, 2008; Jia and Conrad, 2009; Liu et al., 2018). In the organic nutrient environment, AOA participates in the decomposition of organic matter and COM using ammonium obtained from the mineralization of organic matter. This explains why there was a positive correlation between them and their abundance increased with the addition of organic matter; (2) COM makes use of nitrite nitrogen produced by AOA nitrification. Koch et al. (2019) reported that $\mathrm{COM}$ is genetically capable of using nitrite nitrogen, which explains why AOA and COM were positively correlated.

The addition of urea in a mixed nutrient environment increases the content of available soil N, thereby AOA and AOB compete for the ammonium produced by urea hydrolysis. Xue et al. (2016) reported that AOA has more competitive advantages under mixed nutrition conditions compared to $A O B$, which is consistent with our results of a negative correlation between the abundance of $A O A$ and $A O B(R=-0.81, P<0.01)$. Furthermore, $C O M$ still uses the ammonium obtained from the mineralization of organic matter in areas with a low ammonium concentration in the soil.

\section{Conclusions}

This study showed potato fermented fertilizer stimulate the growth and differentiation of soil microorganisms. The two fertilization formulas (potato fermented fertilizer without or with chemical fertilizer) shaped the different soil nutrient environments, thereby changing the niche of $A O A, A O B$, and comammox (including clade $A$ and $B$ ) in the study soil. Soil functional microorganisms $A O A$ and comammox may have a mutually beneficial relationship in an organic nutrient environment shaped by potato fermented fertilizer, which is replaced by a competitive relationship between AOA and $A O B$ in a mixed nutrient environment shaped by potato fertilizer with chemical fertilizer. The results indicated that potato fermented fertilizer provides the necessary nutrients for plant growth, either directly or by stimulating the nitrification process in the soil, increasing yields.

\section{Declarations}




\section{Acknowledgments}

The authors gratefully acknowledge the financial support from the National Key Research and Development Program of China (2021YFD1700803), the Natural Science Foundation of Zhejiang Province (LZ21C030002) and the National Natural Science Foundation of China (41877044).

\section{Compliance with ethical standards}

Conflict of interest: The authors declare no conflict of interest.

Ethical approval: This article does not contain any experiments involving human participants or animals performed by any authors; thus, ethical approval is not necessary

\section{References}

1. Abbas T, Zhang Q, Zou X, Tahir M, Wu D, Jin S, Di H (2020) Soil anammox and denitrification processes connected with $\mathrm{N}$ cycling genes co-supporting or contrasting under different water conditions. Environ Int 140. https://doi.org/10.1016/j.envint.2020.105757

2. Ali W, Nadeem M, Ashiq W, Zaeem M, Gilani SSM, Rajabi-Khamseh S, Pham TH, Kavanagh V, Thomas R, Cheema M (2019) The effects of organic and inorganic phosphorus amendments on the biochemical attributes and active microbial population of agriculture podzols following silage corn cultivation in boreal climate. Sci Rep 9:1-17. https://doi.org/10.1038/s41598-019-53906-8

3. Bae H-S, Morrison E, Chanton JP, Ogram A (2018) Methanogens are major contributors to nitrogen fixation in soils of the Florida Everglades. 84. Applied and environmental microbiologyhttps://doi.org/10.1128/aem.02222-17

4. Bissey LL, Smith JL, Watts RJ (2006) Soil organic matter-hydrogen peroxide dynamics in the treatment of contaminated soils and groundwater using catalyzed H2O2 propagations (modified Fenton's reagent). Water Res 40:2477-2484. https://doi.org/10.1016/j.watres.2006.05.009

5. Cai M, Hu C, Wang X, Zhao Y, Jia W, Sun X, Elyamine AM, Zhao X (2019) Selenium induces changes of rhizosphere bacterial characteristics and enzyme activities affecting chromium/selenium uptake by pak choi (Brassica campestris L. ssp. Chinensis Makino) in chromium contaminated soil. Environ Pollut 249:716-727. https://doi.org/10.1016/j.envpol.2019.03.079

6. Chevalier S, Bouffartigues E, Bodilis J, Maillot O, Lesouhaitier O, Feuilloley MG, Orange N, Dufour A, Cornelis PJF (2017) Structure, function and regulation of Pseudomonas aeruginosa porins. FEMS Microbiol Rev 41:698-722. m.r https://doi.org/10.1093/femsre/fux020

7. Cotto I, Dai Z, Huo L, Anderson CL, Vilardi KJ, ljaz U, Khunjar W, Wilson C, De Clippeleir H, Gilmore K (2020) Long solids retention times and attached growth phase favor prevalence of comammox bacteria in nitrogen removal systems. Water Res 169:115268. https://doi.org/10.1016/j.watres.2019.115268

8. Daims H, Lebedeva EV, Pjevac P, Han P, Herbold C, Albertsen M, Jehmlich N, Palatinszky M, Vierheilig J, Bulaev A (2015a) Complete nitrification by Nitrospira bacteria. Nature 528:504-509. https://doi.org/10.1038/nature16461

9. Fierer N, Lauber CL, Ramirez KS, Zaneveld J, Bradford MA, Knight R (2012) Comparative metagenomic, phylogenetic and physiological analyses of soil microbial communities across nitrogen gradients. The I S M E journal 6:10071017. https://doi.org/10.1038/ismej.2011.159

10. Fontaine S, Bardoux G, Abbadie L, Mariotti AJEL (2010) Carbon input to soil may decrease soil carbon content. Ecol Lett 7:314-320. https://doi.org/10.1111/j.1461-0248.2004.00579.x 
11. Jia Z, Conrad R (2009) Bacteria rather than Archaea dominate microbial ammonia oxidation in an agricultural soil. Environ Microbiol 11:1658-1671. https://doi.org/10.1111/j.1462-2920.2009.01891.x

12. Kakosová E, Hrabák P, Černík M, Novotný V, Czinnerová M, Trögl J, Popelka J, Kuráň P, Zoubková L, Vrtoch L' (2017) Effect of various chemical oxidation agents on soil microbial communities. Chem Eng J 314:257-265. https://doi.org/10.1016/j.cej.2016.12.065

13. Karigar C, Mahesh A, Nagenahalli M, Yun DJJB (2006) Phenol Degradation by Immobilized Cells of Arthrobacter citreus. Biodegradation 17:47-55. https://doi.org/10.1007/s10532-005-3048-y

14. Kaya C, Akram NA, Sürücü A, Ashraf M (2019) Alleviating effect of nitric oxide on oxidative stress and antioxidant defence system in pepper (Capsicum annuum L.) plants exposed to cadmium and lead toxicity applied separately or in combination. Sci Hort 255:52-60. https://doi.org/10.1016/j.scienta.2019.05.029

15. Kim J-G, Park S-J, Damsté JSS, Schouten S, Rijpstra WIC, Jung M-Y, Kim S-J, Gwak J-H, Hong H, Si O-J (2016) Hydrogen peroxide detoxification is a key mechanism for growth of ammonia-oxidizing archaea. Proceedings of the National Academy of Sciences 113, 7888-7893. https://doi.org/10.1073/pnas.1605501113

16. Kits KD, Sedlacek CJ, Lebedeva EV, Han P, Bulaev A, Pjevac P, Daebeler A, Romano S, Albertsen M, Stein LY (2017) Kinetic analysis of a complete nitrifier reveals an oligotrophic lifestyle. Nature 549:269-272. https://doi.org/10.1038/nature23679

17. Koch H, van Kessel MA, Lücker S (2019) Complete nitrification: insights into the ecophysiology of comammox Nitrospira. Appl Microbiol Biotechnol 103:177-189. https://doi.org/10.1007/s00253-018-9486-3

18. Kot AM, Pobiega K, Piwowarek K, Kieliszek M, Błażejak S, Gniewosz M, Lipińska E (2020) Biotechnological methods of management and utilization of potato industry waste-a review. Potato Res 1-17.

https://doi.org/10.1007/s11540-019-09449-6

19. Kurcz A, Błażejak S, Kot AM, Bzducha-Wróbel A (2016) Wykorzystanie odpadów pochodzących z przemysłu rolnospożywczego do produkcji biomasy drożdży paszowych Candida utilis. Postępy Mikrobiologii 55:19-26

20. Leite MF, Pan Y, Bloem J, Berge T, Kuramae H, E.E (2017) Organic nitrogen rearranges both structure and activity of the soil-borne microbial seedbank. Sci Rep 7:1-11. https://doi.org/10.1038/srep42634

21. Li L-J, Zhu-Barker X, Ye R, Doane TA, Horwath WR (2018) Soil microbial biomass size and soil carbon influence the priming effect from carbon inputs depending on nitrogen availability. Soil Biol Biochem 119:41-49.

https://doi.org/10.1016/j.soilbio.2018.01.003

22. Li W, Wang J, Li X, Wang S, Liu W, Shi S, Cao W (2019a) Nitrogen fertilizer regulates soil respiration by altering the organic carbon storage in root and topsoil in alpine meadow of the north-eastern Qinghai-Tibet Plateau. Sci Rep 9:1-13. https://doi.org/10.1038/s41598-019-50142-y

23. Li Y, Fang F, Wei J, Wu X, Cui R, Li G, Zheng F, Tan D (2019b) Humic acid fertilizer improved soil properties and soil microbial diversity of continuous cropping peanut: a three-year experiment. Sci Rep 9:1-9. https://doi.org/10.1038/s41598-019-48620-4

24. Liang J, Zhou Z, Huo C, Shi Z, Cole JR, Huang L, Konstantinidis KT, Li X, Liu B, Luo Z (2018) More replenishment than priming loss of soil organic carbon with additional carbon input. Nat Commun 9:1-9. https://doi.org/10.1038/s41467-018-05667-7

25. Liu H, Li J, Zhao Y, Xie K, Tang X, Wang S, Li Z, Liao Y, Xu J, Di H (2018) Ammonia oxidizers and nitrite-oxidizing bacteria respond differently to long-term manure application in four paddy soils of south of China. Sci Total Environ 633:641-648. https://doi.org/10.1016/j.scitotenv.2018.03.108

26. Liu Y, Fan X, Zhang T, He W, Song F (2020a) Effects of the long-term application of atrazine on soil enzyme activity and bacterial community structure in farmlands in China. Environ Pollut 262:114264.

https://doi.org/10.1016/j.envpol.2020.114264

Page $13 / 18$ 
27. Liu Z, Zhang C, Wei Q, Zhang S, Quan Z, Li M (2020b) Temperature and salinity drive comammox community composition in mangrove ecosystems across southeastern China. Sci Total Environ 742:140456. https://doi.org/10.1016/j.scitotenv.2020.140456

28. Markiewicz M, Przewodowska A, Przewodowski W, Stochła W, Środowiskiem CB, IHAR-PIB ZN (2015) Wykorzystanie chromatografii membranowej do odzyskiwania białek aktywnych biologicznie z odpadów przemysłu skrobiowego. Annual Set The Environment Protection Rocznik Ochrona Srodowiska 17, 1699-1714

29. Mehnaz KR, Corneo PE, Keitel C, Dijkstra FA (2019) Carbon and phosphorus addition effects on microbial carbon use efficiency, soil organic matter priming, gross nitrogen mineralization and nitrous oxide emission from soil. Soil Biol Biochem 134:175-186. https://doi.org/10.1016/j.soilbio.2019.04.003

30. Miedzianka J, Pęksa A, Pokora M, Rytel E, Tajner-Czopek A, Kita A (2014) Improving the properties of fodder potato protein concentrate by enzymatic hydrolysis. Food Chem 159:512-518.

https://doi.org/10.1016/j.foodchem.2014.03.054

31. Noll L, Zhang S, Wanek W (2019) Novel high-throughput approach to determine key processes of soil organic nitrogen cycling: gross protein depolymerization and microbial amino acid uptake. Soil Biol Biochem 130:73-81. https://doi.org/10.1016/j.soilbio.2018.12.005

32. Norton JM, Klotz MG, Stein LY, Arp DJ, Bottomley PJ, Chain PS, Hauser LJ, Land ML, Larimer FW, Shin MWJA (2008) microbiology, e., Complete genome sequence of Nitrosospira multiformis, an ammonia-oxidizing bacterium from the soil environment. Applied and Environmental Microbiology 74, 3559-3572.

https://doi.org/10.1128/AEM.02722-07

33. Nunes-Alves C (2016) Microbial ecology: Do it yourself nitrification. Nat Rev Microbiol 14:61-61. https://doi.org/10.1038/nrmicro.2015.20

34. Prosser Jl, Nicol GW (2008) Relative contributions of archaea and bacteria to aerobic ammonia oxidation in the environment. Environ Microbiol 10:2931-2941. https://doi.org/10.1111/j.1462-2920.2008.01775.x

35. Sakoula D, Koch H, Frank J, Jetten MS, Van Kessel MA, Lücker S (2020) Enrichment and physiological characterization of a novel comammox Nitrospira indicates ammonium inhibition of complete nitrification. The I.S.M.E. journal. 1-15. https://doi.org/10.1038/s41396-020-00827-4

36. Santoro AE (2016) The do-it-all nitrifier. Science 351:342-343. https://doi.org/10.1126/science.aad9839

37. Santos P, Pinhal I, Rainey FA, Empadinhas N, Costa J, Fields B, Benson R, Veríssimo A, Da Costa MSJA, microbiology e (2003) Gamma-proteobacteria Aquicella lusitana gen. nov., sp. nov., and Aquicella siphonis sp. nov. infect protozoa and require activated charcoal for growth in laboratory media. Appl Environ Microbiol 69:65336540. https://doi.org/10.1128/AEM.69.11.6533-6540.2003

38. Sperfeld M, Rauschenbach C, Diekert G, Studenik SJWr (2018) Microbial community of a gasworks aquifer and identification of nitrate-reducing Azoarcus and Georgfuchsia as key players in BTEX degradation. 132:146-157. https://doi.org/10.1016/j.watres.2017.12.040

39. Spieck E, Sass K, Keuter S, Hirschmann S, Spohn M, Indenbirken D, Kop LF, Lücker S, Giaveno AJF (2020) Defining culture conditions for the hidden nitrite-oxidizing bacterium Nitrolancea. Front Microbiol 11:1522. i.m https://doi.org/10.3389/fmicb.2020.01522

40. Tang H, Li C, Xiao X, Shi L, Cheng K, Wen L, Li W (2020) Effects of short-term manure nitrogen input on soil microbial community structure and diversity in a double-cropping paddy field of southern China. Sci Rep 10:1-9. https://doi.org/10.1038/s41598-020-70612-y

41. Van Kessel MA, Speth DR, Albertsen M, Nielsen PH, den Camp HJO, Kartal B, Jetten MS, Lücker S (2015) Complete nitrification by a single microorganism. Nature 528:555-559. https://doi.org/10.1038/nature16459 
42. Wang R-M, Wang Y, Ma G-P, He Y-F, Zhao Y-Q (2009) Efficiency of porous burnt-coke carrier on treatment of potato starch wastewater with an anaerobic-aerobic bioreactor. Chem Eng J 148:35-40.

https://doi.org/10.1016/j.cej.2008.07.028

43. Xu S, Wang B, Li Y, Jiang D, Zhou Y, Ding A, Zong Y, Ling X, Zhang S, Lu H (2020) Ubiquity, diversity, and activity of comammox Nitrospira in agricultural soils. Sci Total Environ 706:135684.

http://doi.org/10.1016/j.scitotenv.2019.135684

44. Xue C, Zhang X, Zhu C, Zhao J, Zhu P, Peng C, Ling N, Shen Q (2016) Quantitative and compositional responses of ammonia-oxidizing archaea and bacteria to long-term field fertilization. Sci Rep 6:1-11. https://doi.org/10.1038/srep28981

45. Yu P, Tang X, Zhang A, Fan G, Liu S (2019) Responses of soil specific enzyme activities to short-term land use conversions in a salt-affected region, northeastern China. Sci Total Environ 687:939-945. https://doi.org/10.1016/j.scitotenv.2019.06.171

46. Zhang Q, Zhu L, Wang J, Xie H, Wang J, Wang F, Sun F (2014) Effects of fomesafen on soil enzyme activity, microbial population, and bacterial community composition. Environ Monit Assess 186:2801-2812. https://doi.org/10.1007/s10661-013-3581-9

47. Zhou H, Zhang Y, Zhang H, Li H (2020) Screening of a strain of degradable protein in potato starch juice and analysis of fermentation products. Food and fermentation industries 1-12. https://doi.org/10.13995/j.cnki.11$1802 /$ ts.025776

48. Fowler SJ, Palomo A, Dechesne A, Mines PD, Smets BF J.E.m., 2018. Comammox Nitrospira are abundant ammonia oxidizers in diverse groundwater-fed rapid sand filter communities. 20,1002-1015

49. Francis CA, Roberts KJ, Beman JM, Santoro AE, Oakley BBJP (2005) Ubiquity and diversity of ammonia-oxidizing archaea. in water columns and sediments of the ocean 102:14683-14688. o.t.N.A.o.S

50. He Jz, Shen Jp, Zhang Lm, Zhu Yg, Zheng Ym, Xu Mg, Di HJEm (2007) Quantitative analyses of the abundance and composition of ammonia-oxidizing bacteria and ammonia-oxidizing archaea of a Chinese upland red soil under long-term fertilization practices. 9:2364-2374

51. Pester M, Maixner F, Berry D, Rattei T, Koch H, Lücker S, Nowka B, Richter A, Spieck E, Lebedeva EJEm (2014) NxrB encoding the beta subunit of nitrite oxidoreductase as functional and phylogenetic marker for nitrite-oxidizing $\mathrm{N}$ itrospira. 16:3055-3071

52. Yoshida M, Ishii S, Otsuka S, Senoo KJM, Environments (2009) nirK-harboring denitrifiers are more responsive to denitrification-inducing conditions in rice paddy soil than nirS-harboring bacteria.0912180141-0912180141

\section{Figures}

\section{Figure 1}

Soil enzyme activities under different depth and application amount of potato wastewater. (a) Soil enzyme activities of PW1, PW2, PW3 treatments at topsoil. (b) Soil enzyme activities of PW1, PW2, PW3 treatments at deep soil. (c) Soil enzyme activities of PWF1, PWF2, PWF3 treatments at topsoil. (d) Soil enzyme activities of PWF1, PWF2, PWF3 treatments at deep soil.

Note: Different lowercase letters indicate significant difference in activity of the same enzyme among the different treatments $(n=3, P<0.05)$.

Page 15/18 


\section{Figure 2}

Principal coordinate analysis of $\beta$ diversity of every treatment. (a) PW1, PW2, PW3 treatments at topsoil. (b) PW1, PW2, PW3 treatments at deep soil. (c) PWF1, PWF2, PWF3 treatments at topsoil. (d) PWF1, PWF2, PWF3 treatments at deep soil.

\section{Figure 3}

Box line diagram and volcano map of differentially expressed OTU between CK and PW1(a), PW2(b), PW3(c), between CK and PWF1 (d), PWF2(e), PWF3(f) at topsoils. Differentially expressed OTU between CK and PW1(g), PW2(h), PW3(i), between CK and PWF1 (j), PWF2(k), PWF3(I) at deep soils.

\section{Figure 4}

Analysis of covariance networks of treatments CK, PW, PWF. (a) networks of treatments CK, PW, PWF. (b) Relative species abundance at phylum in network. (c) Topological properties of covariance networks of treatment CK, PW, PWF. 

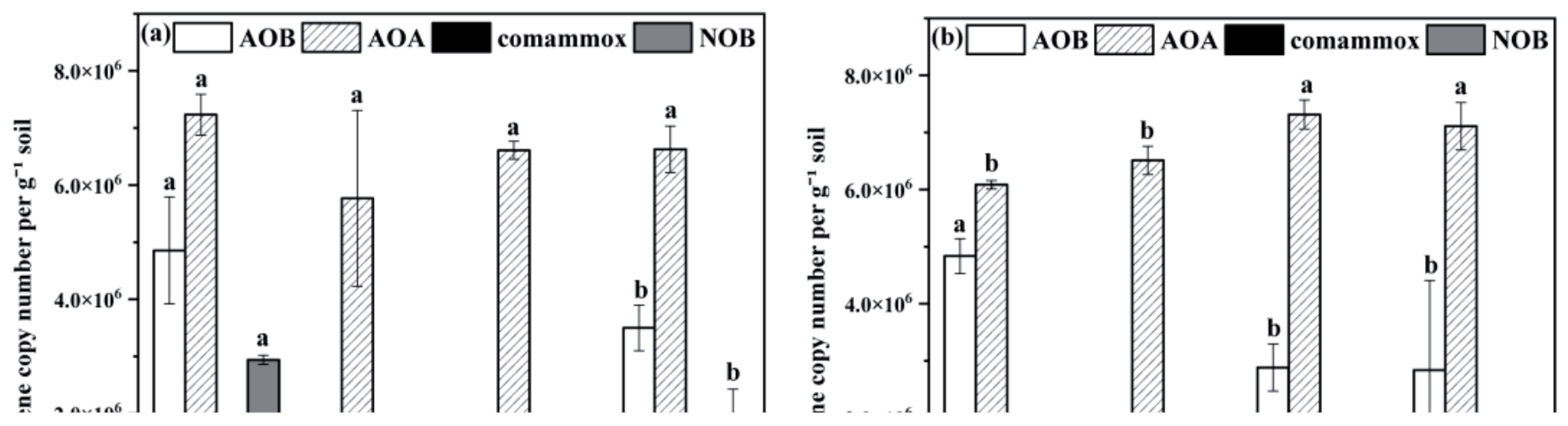

Figure 5

Copies of AOA, AOB, NOB and comammox under different fertilizer treatments. (a) PW1, PW2, PW3 treatments at topsoil. (b) PW1, PW2, PW3 treatments at deep soil. (c) PWF1, PWF2, PWF3 treatments at topsoil. (d) PWF1, PWF2, PWF3 treatments at deep soil.

Note: Different lowercase letters indicate a significant difference in the same gene among the different treatments $(n=3$, $P<0.05)$.

\section{Figure 6}

The heat maps of correlations between environmental factors and nitrogen cycling functional genes. (a) PW1, PW2, PW3 treatments at deep soil. (b) PWF1, PWF2, PWF3 treatments at deep soil.

Note: $* \star \star$ indicate a significant difference $\mathrm{P}<0.01$; $*$ indicate a significant difference $\mathrm{P}<0.05$. 


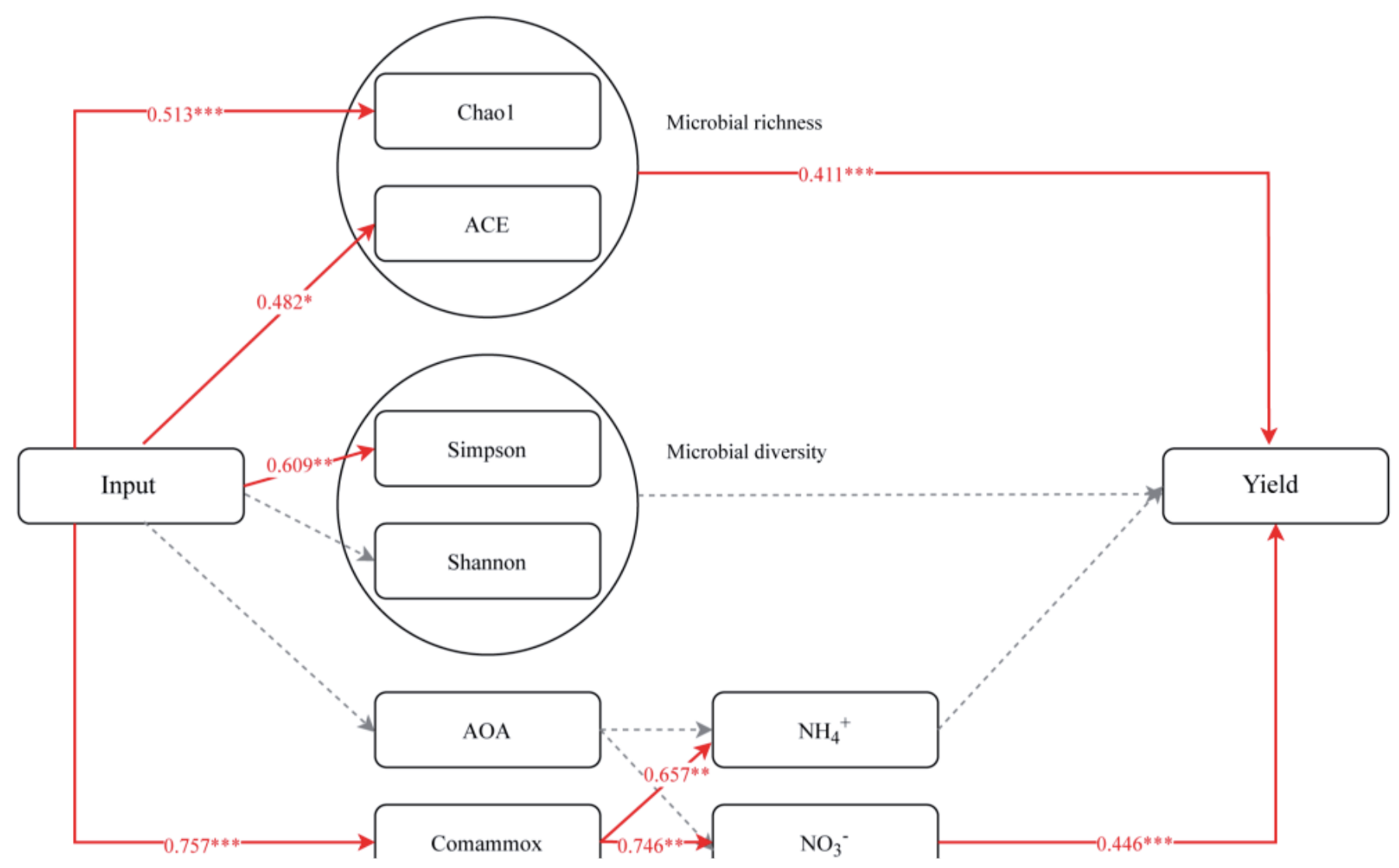

Figure 7

Structural equation modelling between DPW application rate and yield.

\section{Supplementary Files}

This is a list of supplementary files associated with this preprint. Click to download.

- 1GraphicalAbstracts.jpg

- Supplementarymaterial.docx 